International Journal of Pure and Applied Mathematics

Volume 102 No. 3 2015, 515-525

ISSN: 1311-8080 (printed version); ISSN: 1314-3395 (on-line version)

url: http://www.ijpam.eu

doi: http://dx.doi.org/10.12732/ijpam.v102i3.9

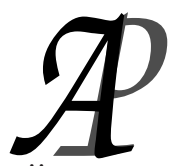

ijpam.eu

\title{
INVARIANT CORE FOR DOUBLE SEQUENCES IN NON-ARCHIMEDEAN FIELDS
}

\author{
S. Sangeetha ${ }^{1}$, V. Srinivasan ${ }^{2}$ \\ ${ }^{1,2}$ Department of Mathematics \\ SRM University \\ Kattankulathur, Chennai, 603 203, INDIA
}

\begin{abstract}
In this paper, $K$ denotes a complete, non-trivially valued, nonarchimedean field. The entries of sequences, series and infinite matrices are in $K$. In the present paper, we prove the Knopp's core theorem for double sequences in $K$ and also the necessary and sufficient conditions for the core to be invariant under the four dimensional matrix transformation in such fields.
\end{abstract}

AMS Subject Classification: 40A05, 40C05, 46S10

Key Words: core of a double sequence, regular matrix, Knopp's core theorem, invariant core, non-archimedean fields

\section{Introduction}

Throughout this paper $K$ denotes a complete, non-trivially valued, non-archimedean field, where the non-archimedean valuation satisfies the following axioms:

(i) $|x| \geq 0$ and $|x|=0$ iff $x=0$.

(ii) $|x y|=|x||y|$.

Received: April 2, 2015

(C) 2015 Academic Publications, Ltd.

$\S$ Correspondence author url: www.acadpubl.eu 
(iii) $|x+y| \leq \max \{|x|,|y|\}$ for all $x, y \in K$.

For analysis in the classical case a general reference is [3], while for analysis in non-archimedean fields, a general reference is [2].

Patterson [8] has proved the Invariant core theorem for double sequences in classical case. We prove here its analogue for double sequences in a complete, non-trivially valued, non-archimedean field.

For a given infinite matrix $A=\left(a_{m n k l}\right)$, we define

$$
A x=(A x)_{m n}=\sum_{k=1, l=1}^{\infty, \infty} a_{m n k l} x_{k l}, \quad m, n=1,2,3, \ldots,
$$

assuming that the series on the right converge and $\left\{(A x)_{m n}\right\}$ is called the $A$ transform of the double sequence $x=\left\{x_{k l}\right\}$.

If, whenever $\left\{x_{m n}\right\}$ converges to a limit, $\left\{(A x)_{m n}\right\}$ converges to the same limit, then the matrix $A=\left(a_{m n k l}\right)$ is said to be regular.

Definition 1. Let $C_{n}(x), n=1,2,3, \ldots$ be the smallest closed convex set containing all points $x_{k l}$ for $k, l>n$. We define the core of the double sequence $x=\left\{x_{k l}\right\}$ as

$$
\mathcal{K}(x)=\bigcap_{n=1}^{\infty} C_{n}(x) .
$$

Also define $C_{r}(u)=\left\{u^{\prime} \in K|| u^{\prime}-u \mid \leq r\right\}$, where $C(0)=\{u \in K|| u \mid \leq 1\}$.

In this context, we refer to Silvermann-Toeplitz theorem for double sequences and series in non-archimedean fields [6].

Definition 2. Let $\left\{x_{k l}\right\}$ be a double sequence in $K$ and $x \in K$. We say that $\lim _{k+l \rightarrow \infty} x_{k l}=x$ if for each $\epsilon>0$, the set $\left\{(k, l) \in \mathbb{N}^{2}:\left|x-x_{k l}\right| \geq \epsilon\right\}$ is finite. In such a case we say that $x$ is the limit of $\left\{x_{k l}\right\}$.

Definition 3. Let $\left\{x_{k l}\right\}$ be a double sequence in $K$ and $s \in K$. We say that

$$
s=\sum_{k=1, l=1}^{\infty, \infty} x_{k l},
$$

if $s=\lim _{k+l \rightarrow \infty} s_{k l}$ where $s_{k l}=\sum_{i=1, j=1}^{k, l} x_{i j}, k, l=1,2, \ldots$.

Remark 4. If $\lim _{k+l \rightarrow \infty} x_{k l}=x$, then the sequence $\left\{x_{k l}\right\}$ is automatically bounded. 
Lemma 5. $\lim _{k+l \rightarrow \infty} x_{k l}=x$ if and only if

(i) $\lim _{l \rightarrow \infty} x_{k l}=x, k=1,2, \ldots$

(ii) $\lim _{k \rightarrow \infty} x_{k l}=x, l=1,2, \ldots$ and

(iii) for each $\epsilon>0$ there exists an $N \in \mathbb{N}$ such that $\left|x-x_{k l}\right|<\epsilon$, for all $k, l \geq N$, which we write as $\lim _{k, l \rightarrow \infty} x_{k l}=x$.

Lemma 6. $\lim _{k+l \rightarrow \infty} s_{k l}$ exists if and only if $\lim _{k+l \rightarrow \infty} x_{k l}=0$.

Theorem 7. In order that whenever a sequence $\left\{x_{m n}\right\}$ has a limit $x$, $\sum_{k=1, l=1}^{\infty, \infty} a_{m n k l} x_{k l}$, shall converge and $\lim _{m+n \rightarrow \infty} \sum_{k=1, l=1}^{\infty, \infty} a_{m n k l} x_{k l}=x$ i.e., for $A=$ $\left(a_{m n k l}\right)$ to be regular it is necessary and sufficient that

(a) $\lim _{m+n \rightarrow \infty} a_{m n k l}=0, k, l=1,2, \ldots$,

(b) $\lim _{m+n \rightarrow \infty} \sum_{k=1, l=1}^{\infty, \infty} a_{m n k l}=1$,

(c) $\lim _{m+n \rightarrow \infty} \sup _{k \geq 1}\left|a_{m n k l}\right|=0, l=1,2, \ldots$,

(d) $\lim _{m+n \rightarrow \infty} \sup _{l \geq 1}\left|a_{m n k l}\right|=0, k=1,2, \ldots$,

(e) $\sup _{m, n, k, l}\left|a_{m n k l}\right|<\infty$.

\section{Main Result}

Theorem 8. An infinite matrix $A=\left(a_{m n k l}\right), a_{m n k l} \in K$ is such that $\mathcal{K}(A x) \subset \mathcal{K}(x)$ for a double sequence $x=\left\{x_{k l}\right\}$ iff $A$ is regular and satisfies

$$
\lim _{m+n \rightarrow \infty} \sup _{k, l \geq 1}\left|a_{m n k l}\right|=1 .
$$


Proof. Necessary part:

Let $x=\left\{x_{k l}\right\}$ be a double sequence converging to $L$ in $K$ such that $A=\left(a_{m n k l}\right)$ is regular and satisfies (1). If $v$ is any point of $\mathcal{K}(A x)$, then

$$
\begin{aligned}
|v-L| & \leq \lim _{m+n \rightarrow \infty} \sup _{k, l \geq 1}\left|(A x)_{m n}-L\right|, \quad L \in K . \\
& =\lim _{m+n \rightarrow \infty} \sup _{k, l \geq 1}\left|\sum_{k, l=1}^{\infty, \infty} a_{m n k l} x_{k l}-L\right|, \quad L \in K . \\
& =\lim _{m+n \rightarrow \infty} \sup _{k, l \geq 1}\left|\sum_{k, l=1}^{\infty, \infty} a_{m n k l} x_{k l}-\sum_{k, l=1}^{\infty, \infty} a_{m n k l} L\right| \quad \text { (using (b) of Theorem 7) } \\
& =\lim _{m+n \rightarrow \infty} \sup _{k, l \geq 1}\left|\sum_{k, l=1}^{\infty, \infty} a_{m n k l}\left(x_{k l}-L\right)\right| \\
& \leq \lim _{m+n \rightarrow \infty} \sup _{k, l \geq 1}\left|x_{k l}-L\right| \operatorname{using}(i) \\
i . e .,|v-L| & \leq \lim _{m+n \rightarrow \infty} \sup _{k, l \geq 1}\left|x_{k l}-L\right| \\
\Rightarrow v \in \mathcal{K}(x) . &
\end{aligned}
$$

Thus, $v \in \mathcal{K}(A x)$ implies $v \in \mathcal{K}(x)$ gives, $\mathcal{K}(A x) \subset \mathcal{K}(x)$.

Sufficient part:

Conversely, if $\mathcal{K}(A x) \subset \mathcal{K}(x)$, then it is clear that $\left\{(A x)_{m n}\right\}$ is a convergent sequence and has a limit in $\mathcal{K}(x)$ Therefore, $A$ is regular. (see [4]).

Now to Prove (i), we shall suppose that (i) does not hold

$$
\lim _{m+n \rightarrow \infty} \sup _{k, l \geq 1}\left|a_{m n k l}\right| \neq 1
$$

and arrive at a contradiction.

Let if possible $\lim _{m+n \rightarrow \infty} \sup _{k, l \geq 1}\left|a_{m n k l}\right|>1$.

Since $K$ is non-trivially valued, there exists $\pi \in K$ such that $0<\rho=|\pi|<$ 1.

Let us choose $m_{1}=n_{1}=1$. Using (a), (b) of Theorem 7, choose $m_{2}+n_{2}>$ $m_{1}+n_{1}$, there exists $\epsilon>0$, such that

$$
\begin{aligned}
& \sup _{1 \leq k+l \leq m_{1}+n_{1}}\left|a_{m_{2} n_{2} k l}\right|<\frac{\epsilon}{8} \\
& \sup _{k+l \geq 1}\left|a_{m_{2} n_{2} k l}\right|>\frac{\epsilon}{\rho} \text { and }
\end{aligned}
$$


$\sup _{k+l \geq m_{2}+n_{2}}\left|a_{m_{2} n_{2} k l}\right|<\frac{\epsilon}{8}$

Inductively choose $m_{p}+n_{p}>m_{p-1}+n_{p-1}$ such that,

$$
\begin{gathered}
\sup _{1 \leq k+l \leq m_{p-1}+n_{p-1}}\left|a_{m_{p} n_{p} k l}\right|<\frac{\epsilon}{8} \\
\sup _{k+l \geq 1}\left|a_{m_{p} n_{p} k l}\right|>\frac{\epsilon}{\rho} \text { and } \\
\sup _{k+l>m_{p}+n_{p}}\left|a_{m_{p} n_{p} k l}\right|<\frac{\epsilon}{8}
\end{gathered}
$$

In view of $(2),(3),(4)$, we have

$$
\begin{aligned}
\sup _{m_{p-1}+n_{p-1}<k+l \leq m_{p}+n_{p}}\left|a_{m_{p} n_{p} k l}\right| & >\frac{\epsilon}{\rho}-\frac{\epsilon}{8}-\frac{\epsilon}{8} \\
& >\frac{\epsilon}{\rho}-\frac{\epsilon}{8 \rho}-\frac{\epsilon}{8 \rho}=\frac{3 \epsilon}{4 \rho}
\end{aligned}
$$

Thus, there exist $k_{p}$ and $l_{p}, m_{p-1}+n_{p-1}<k_{p}+l_{p} \leq m_{p}+n_{p}$ such that $\left|a_{m_{p} n_{p} k_{p} l_{p}}\right|>\frac{3 \epsilon}{4 \rho}$.

Now, define the sequence $\left\{x_{k l}\right\}$ as follows:

$$
x_{k l}= \begin{cases}\rho, & \text { if } k=k_{p}, l=l_{p}, p=1,2, \ldots \\ 0, & \text { otherwise }\end{cases}
$$

For this sequence $x, \lim _{k+l \rightarrow \infty} x_{k l}=0$ implies $\mathcal{K}(x) \subset C(0)$. Now,

$$
\begin{aligned}
& \left|(A x)_{m_{p} n_{p}}\right|=\left|\sum_{k=1, l=1}^{\infty, \infty} a_{m_{p} n_{p} k l} x_{k l}\right| \\
& \geq\left|\sum_{k+l=\left(m_{p-1}+n_{p-1}\right)+1}^{m_{p}+n_{p}} a_{m_{p} n_{p} k l} x_{k l}\right|-\left|\sum_{k+l=1}^{m_{p-1}+n_{p-1}} a_{m_{p} n_{p} k l} x_{k l}\right| \\
& \quad-\left|\sum_{k+l=\left(m_{p}+n_{p}\right)+1}^{\infty} a_{m_{p} n_{p} k l} x_{k l}\right| \\
& >\left|a_{m_{p} n_{p} k_{p} l_{p}}\right|\left|x_{k_{p} l_{p}}\right|-\sup _{1 \leq k+l \leq m_{p-1}+n_{p-1}}\left|a_{m_{p} n_{p} k l}\right|-a_{k+l>m_{p}+n_{p}} \sup _{k m_{p} n_{p} k l} \mid \\
& >\frac{3 \epsilon}{4 \rho} \cdot \rho-\frac{\epsilon}{8}-\frac{\epsilon}{8}=\frac{\epsilon}{2}
\end{aligned}
$$


$\Rightarrow\left|(A x)_{m_{p} n_{p}}\right|>\frac{\epsilon}{2}$

Therefore, $\lim _{m+n \rightarrow \infty}(A x)_{m n}=0$ does not hold, which implies that $(A x)_{m n} \notin C(0)$.

i.e., $\mathcal{K}(A x) \not \subset C(0)$, which is a contradiction.

This completes the proof of the theorem.

Theorem 9. The core of a double sequence $\left\{x_{k l}\right\}$ is invariant under the regular A-transformation iff

(i) $\lim _{m+n \rightarrow \infty} \sup _{k, l \geq 1}\left|a_{m n k l}\right|=1$.

(ii) for every given pair of index sequences $\left(k_{j}, l_{j}\right), i, j=1,2, \ldots$ there exist a pair of index sequences $\left(m_{p}, n_{q}\right), p, q=1,2, \ldots$ such that

$$
\lim _{m_{p}+n_{q} \rightarrow \infty} \sum_{i, j=1}^{\infty} a_{m_{p} n_{q} k_{i} l_{j}}=1 .
$$

Proof. Necessary part:

Assume $\mathcal{K}(A x)=\mathcal{K}(x)$. Then by Theorem 8 condition (i) holds.

The proof of condition (ii) is as follows.

Let $\left(k_{i}, l_{j}\right)$ for $i, j=1,2, \ldots$ be given pair of index sequences and define $\left\{x_{k l}\right\}$ as follows:

$$
x_{k l}= \begin{cases}1, \text { if } k=k_{i} \text { and } l=l_{j} & \\ 0, & \text { otherwise. }\end{cases}
$$

Then we find that if there exists an $N$ such that $x_{k l} \neq 0$ whenever $k, l>N$, then $\mathcal{K}(A x)=\mathcal{K}(x)=1$. This implies that there exist a double subsequence of $\left\{(A x)_{m n}\right\}$ with limit 1. i.e., there exist a pair of index sequences $\left(m_{p}, n_{q}\right)$, $p, q=1,2, \ldots$ such that

$$
\lim _{m_{p}+n_{q} \rightarrow \infty} \sum_{i, j=1}^{\infty} a_{m_{p} n_{q} k_{i} l_{j}}=1 .
$$

This completes the necessary part of this theorem.

\section{Sufficient part:}

Let $\left\{x_{k_{i} l_{j}}\right\}$ be a subsequence of $\left\{x_{k l}\right\}$ such that

$$
\lim _{i+j \rightarrow \infty} x_{k_{i} l_{j}}=\alpha
$$


Also, let $\left(k_{i}, l_{j}\right)$ for $i, j=1,2, \ldots$ denote the pair of indices from $\left\{x_{k l}\right\}$ used in the construction of $\left\{(A x)_{m n}\right\}$ and let $\left.\left(\bar{k}_{i}, \bar{l}_{j}\right)\right)$ for $i, j=1,2, \ldots$ be the remaining pair of indices of $\left\{x_{k l}\right\}$.

Let us define $\left\{w_{k l}\right\}$ and $\left\{\bar{w}_{k l}\right\}$ as follows:

$$
w_{k l}= \begin{cases}x_{k_{i} l_{j}}, \text { if } k=k_{i} \text { and } l=l_{j} & \\ 0, & \text { otherwise. }\end{cases}
$$

and

$$
\bar{w}_{k l}= \begin{cases}x_{\bar{k}_{i} \bar{l}_{j}}, \text { if } k=\bar{k}_{i} \text { and } l=\bar{l}_{j} & \\ 0, & \text { otherwise. }\end{cases}
$$

Note that $\left\{x_{k l}\right\}=\left\{w_{k l}\right\}+\left\{\bar{w}_{k l}\right\}$ which gives the following:

$$
\begin{aligned}
(A x)_{m n} & =\sum_{k=1, l=1}^{\infty, \infty} a_{m n k l} x_{k l} \\
& =\sum_{k=1, l=1}^{\infty, \infty} a_{m n k l} w_{k l}+\sum_{k=1, l=1}^{\infty, \infty} a_{m n k l} \bar{w}_{k l} \\
& =\sum_{i=1, j=1}^{\infty, \infty} a_{m n k_{i} l_{j}} x_{k_{i} l_{j}}+\sum_{i=1, j=1}^{\infty, \infty} a_{m n \bar{k}_{i} \bar{l}_{j}} x_{\bar{k}_{i} \bar{l}_{j}}
\end{aligned}
$$

Let $\left(m_{p}, n_{q}\right)$ be the pair of index sequence defined earlier such that

$$
(A x)_{m_{p} n_{q}}=\sum_{i=1, j=1}^{\infty, \infty} a_{m_{p} n_{q} k_{i} l_{j}} x_{k_{i} l_{j}}+\sum_{i=1, j=1}^{\infty, \infty} a_{m_{p} n_{q} \bar{k}_{i} \bar{l}_{j}} x_{\bar{k}_{i} \bar{l}_{j}}
$$

Since $\lim _{i+j \rightarrow \infty} x_{k_{i} l_{j}}=\alpha$, if $\epsilon>0$, we can choose sufficiently large $s, t$ such that $x_{k_{i} l_{j}}=\alpha+\epsilon_{k_{i} l_{j}}$ with $\left|\epsilon_{k_{i} l_{j}}\right|<\frac{\epsilon}{16}$, whenever, $i+j>s+t$.

Then,

$$
\begin{aligned}
(A x)_{m_{p} n_{q}}-\alpha=\left[\sum_{i=1, j=1}^{\infty, \infty} a_{m_{p} n_{q} k_{i} l_{j}} x_{k_{i} l_{j}}+\sum_{i=1, j=1}^{\infty, \infty} a_{m_{p} n_{q} \bar{k}_{i} \bar{l}_{j}} x_{\bar{k}_{i} \bar{l}_{j}}\right]^{-\alpha} \\
=\left[\sum_{i=1, j=1}^{s, t} a_{m_{p} n_{q} k_{i} l_{j}} x_{k_{i} l_{j}}+\sum_{i=1, j=t+1}^{s, \infty} a_{m_{p} n_{q} k_{i} l_{j}} x_{k_{i} l_{j}}+\sum_{i=s+1, j=1}^{\infty, t} a_{m_{p} n_{q} k_{i} l_{j} x_{k_{i} l_{j}}}\right. \\
\left.\quad+\sum_{i=s+1, j=t+1}^{\infty, \infty} a_{m_{p} n_{q} k_{i} l_{j}} x_{k_{i} l_{j}}+\sum_{i=1, j=1}^{\infty, \infty} a_{m_{p} n_{q} \bar{k}_{i} \bar{l}_{j}} x_{\bar{k}_{i} \bar{l}_{j}}\right]^{-\alpha}
\end{aligned}
$$




$$
\begin{aligned}
= & {\left[\sum_{i=1, j=1}^{s, t} a_{m_{p} n_{q} k_{i} l_{j}} x_{k_{i} l_{j}}+\sum_{i=1, j=t+1}^{s, \infty} a_{m_{p} n_{q} k_{i} l_{j}} x_{k_{i} l_{j}}+\sum_{i=s+1, j=1}^{\infty, t} a_{m_{p} n_{q} k_{i} l_{j}} x_{k_{i} l_{j}}\right.} \\
& +\sum_{i=s+1, j=t+1}^{\infty, \infty} a_{m_{p} n_{q} k_{i} l_{j}}\left(\alpha+\epsilon_{k_{i} l_{j}}\right)+\sum_{i=1, j=1}^{\infty, \infty} a_{m_{p} n_{q} \bar{k}_{i} \bar{l}_{j}} x_{\bar{k}_{i} \bar{l}_{j}}-\alpha \\
= & \sum_{i=1, j=1}^{s, t} a_{m_{p} n_{q} k_{i} l_{j}} x_{k_{i} l_{j}}+\sum_{i=1, j=t+1}^{s, \infty} a_{m_{p} n_{q} k_{i} l_{j}} x_{k_{i} l_{j}}+\sum_{i=s+1, j=1}^{\infty, t} a_{m_{p} n_{q} k_{i} l_{j}} x_{k_{i} l_{j}} \\
& +\sum_{i=s+1, j=t+1}^{\infty, \infty} a_{m_{p} n_{q} k_{i} l_{j}} \epsilon_{k_{i} l_{j}}+\alpha\left(\sum_{i=s+1, j=t+1}^{\infty, \infty} a_{m_{p} n_{q} k_{i} l_{j}}-1\right) \\
& +\sum_{i=1, j=1}^{\infty, \infty} a_{m_{p} n_{q} \bar{k}_{i} \bar{l}_{j}} x_{\bar{k}_{i} \bar{l}_{j}}
\end{aligned}
$$

Since $\left\{x_{k l}\right\}$ is bounded, there exists an $M$ such that $\left|x_{k l}\right|<M$. Therefore, the following holds:

$$
\begin{aligned}
& \left|(A x)_{m_{p} n_{q}}-\alpha\right| \\
& \leq\left|\sum_{i=1, j=1}^{s, t} a_{m_{p} n_{q} k_{i} l_{j}} x_{k_{i} l_{j}}\right|+\left|\sum_{i=1, j=t+1}^{s, \infty} a_{m_{p} n_{q} k_{i} l_{j}} x_{k_{i} l_{j}}\right|+\left|\sum_{i=s+1, j=1}^{\infty, t} a_{m_{p} n_{q} k_{i} l_{j}} x_{k_{i} l_{j}}\right| \\
& \quad+\left|\sum_{i=s+1, j=t+1}^{\infty, \infty} a_{m_{p} n_{q} k_{i} l_{j}} \epsilon_{k_{i} l_{j}}\right|+|\alpha|\left|\sum_{i=s+1, j=t+1}^{\infty, \infty} a_{m_{p} n_{q} k_{i} l_{j}}-1\right| \\
& \quad+\left|\sum_{i=1, j=1}^{\infty, \infty} a_{m_{p} n_{q} \bar{k}_{i} \bar{l}_{j}} x_{\bar{k}_{i} \bar{l}_{j}}\right|
\end{aligned}
$$

Therefore, (e) of Theorem 7 implies that we can choose $N_{1}$ such that, whenever $m_{p}+n_{q}>N_{1}$,

$$
\begin{aligned}
& \sup _{1 \leq i+j \leq s+t}\left|a_{m_{p} n_{q} k_{i} l_{j}}\right|<\frac{\epsilon}{16 M}, \\
& \sup _{i>s}\left|a_{m_{p} n_{q} k_{i} l_{j}}\right|<\frac{\epsilon}{16 M}, j=1,2, \ldots, t \text { and } \\
& \sup _{j>t}\left|a_{m_{p} n_{q} k_{i} l_{j}}\right|<\frac{\epsilon}{16 M}, i=1,2, \ldots, s .
\end{aligned}
$$


We also have from (ii)

$$
(A x)_{m_{p} n_{q}}=\sum_{i=1, j=1}^{s, t} a_{m_{p} n_{q} k_{i} l_{j}}+\sum_{i=s+1, j=t+1}^{\infty, \infty} a_{m_{p} n_{q} k_{i} l_{j}}
$$

And if $\alpha \neq 0$, we determine $N_{2}>N_{1}$, so that $\left|(A x)_{m_{p} n_{q}}-1\right| \leq \frac{\epsilon}{16|\alpha|}$ for $m_{p}+n_{q}>N_{2}$. Then from (6),

$$
\begin{aligned}
& \sum_{i=s+1, j=t+1}^{\infty, \infty} a_{m_{p} n_{q} k_{i} l_{j}} \\
& =(A x)_{m_{p} n_{q}}-\sum_{i=1, j=1}^{s, t} a_{m_{p} n_{q} k_{i} l_{j}}-\sum_{i=1, j=t+1}^{s, \infty} a_{m_{p} n_{q} k_{i} l_{j}}-\sum_{i=s+1, j=1}^{\infty, t} a_{m_{p} n_{q} k_{i} l_{j}} \\
& \left|\sum_{i=s+1, j=t+1}^{\infty, \infty} a_{m_{p} n_{q} k_{i} l_{j}}-1\right| \\
& =\left|(A x)_{m_{p} n_{q}}-1-\sum_{i=1, j=1}^{s, t} a_{m_{p} n_{q} k_{i} l_{j}}-\sum_{i=1, j=t+1}^{s, \infty} a_{m_{p} n_{q} k_{i} l_{j}}-\sum_{i=s+1, j=1}^{\infty, t} a_{m_{p} n_{q} k_{i} l_{j}}\right| \\
& \leq\left|(A x)_{m_{p} n_{q}}-1\right|+\left|\sum_{i=1, j=1}^{s, t} a_{m_{p} n_{q} k_{i} l_{j}}\right|+\left|\sum_{i=1, j=t+1}^{s, \infty} a_{m_{p} n_{q} k_{i} l_{j}}\right| \\
& +\left|\sum_{i=s+1, j=1}^{\infty, t} a_{m_{p} n_{q} k_{i} l_{j}}\right| \\
& \leq\left|(A x)_{m_{p} n_{q}}-1\right|+\sup _{1 \leq i+j \leq s+t}\left|a_{m_{p} n_{q} k_{i} l_{j}}\right|+\sup _{j>t}\left|a_{m_{p} n_{q} k_{i} l_{j}}\right|+\sup _{i>s}\left|a_{m_{p} n_{q} k_{i} l_{j}}\right| \\
& \leq \frac{\epsilon}{4|\alpha|}
\end{aligned}
$$

Also, by (i) and (ii), there exists an $N_{3}>N_{2}$ such that

$$
\sup _{i+j>s+t}\left|a_{m_{p} n_{q} k_{i} l_{j}}\right|<1 \text { for } m_{p}+n_{q}>N_{3} .
$$

Now from $(5)$, $\left|(A x)_{m_{p} n_{q}}-\alpha\right| \leq \frac{\epsilon}{2}+M \sup _{i+j \geq 1}\left|a_{m_{p} n_{q} \bar{k}_{i} \bar{l}_{j}}\right|$, if $\alpha \neq 0$. 
$\left|(A x)_{m_{p} n_{q}}\right| \leq \frac{\epsilon}{4}+M \sup _{i+j \geq 1}\left|a_{m_{p} n_{q} \bar{k}_{i} \bar{l}_{j}}\right|$, if $\alpha=0$.

There exists $N_{4}$ such that,

$$
\sup _{i+j \geq 1}\left|a_{m_{p} n_{q} \bar{k}_{i} \bar{l}_{j}}\right| \leq \frac{\epsilon}{2 M}
$$

Therefore, for $m_{p}+n_{q}>N_{4}$, we have,

$$
\left|(A x)_{m_{p} n_{q}}-\alpha\right| \leq \epsilon \text { if } \alpha \neq 0,
$$

and

$$
\begin{gathered}
\left|(A x)_{m_{p} n_{q}}\right| \leq \frac{3 \epsilon}{4} \text { if } \alpha=0 . \\
\Rightarrow \lim _{m+n \rightarrow \infty}(A x)_{m n}=\alpha .
\end{gathered}
$$

$\therefore \alpha \in \mathcal{K}(x)$ implies $\alpha \in \mathcal{K}(A x)$. Hence $\mathcal{K}(x) \subset \mathcal{K}(A x)$.

By Theorem 8 , we conclude that $\mathcal{K}(A x)=\mathcal{K}(x)$.

This completes the proof of the theorem.

\section{References}

[1] H.S. Allen, T-transformations which leave the core of every bounded sequence invariant, J. Lond. Math. Soc., 19 (1944), 42-46.

[2] G. Bachman, Introduction to p-adic numbers and valuation theory, Academic Press, New York (1964).

[3] G.H. Hardy, Divergent Series, Oxford Univ. Press, London (1949).

[4] I.J. Maddox, Some anologues of Knopp's core Theorem, Int. J. Math. Math. Sci., 2, No. 4 (1979), 605-614.

[5] P.N. Natarajan, On the core of a sequence over valued fields, J. Indian Math. Soc., 55 (1990) 189-198.

[6] P.N. Natarajan, V. Srinivasan, Silvermann-Toeplitz theorem for double sequences and series and its application to Norlund means in nonarchimedean fields, Annales Mathematiques Blaise Pascal, 9 (2002), 85100.

[7] Richard F. Patterson, Double sequence core Theorems, Int. J. Math. Math. Sci., 22, No. 4 (1999), 785-793. 
[8] Richard F. Patterson, Invariant core theorems for Double sequences, Southeast Asian Bulletin of Mathematics, 24 (2000), 421-429.

[9] G.M. Robison, Divergent double sequences and series, Trans. Amer. Math. Soc., 28 (1926), 50-73. 
